\title{
Magnetic-divertor stabilization of an axisymmetric plasma with anisotropic temperature
}

\author{
Y. Sasagawa, I. Katanuma, ${ }^{\text {a) }}$ Y. Mizoguchi, and T. Cho \\ Plasma Research Center, University of Tsukuba, Tsukuba, Ibaraki 305-8577, Japan \\ V. P. Pastukhov ${ }^{\text {b) }}$ \\ RRC “Kurchatov Institute," Kurchatov Square, 1, 123182 Moscow, Russia
}

(Received 10 August 2006; accepted 8 November 2006; published online 18 December 2006)

\begin{abstract}
Magnetohydrodynamic stabilization of an axisymmetric mirror plasma with a magnetic divertor is studied. An equation is found for the flute modes, which includes the stabilizing influence of ion temperature anisotropy and nonparaxial magnetic fields, as well as a finite ion Larmor radius. It is shown that if the density profile is sufficiently gentle, then the nonparaxial configuration can stabilize all modes as long as ion temperature is radially uniform. This can be demonstrated even when the density vanishes on the separatrix and even for small ion Larmor radii. It is found, however, that the ion temperature gradient makes the unstable region wider; high ion temperature is required to stabilize the flute mode. (C) 2006 American Institute of Physics.
\end{abstract}

[DOI: 10.1063/1.2402912]

\section{INTRODUCTION}

In a traditional tandem mirror system ${ }^{1,2}$ the interchange modes are suppressed with the help of the nonaxisymmetric minimum-B mirror cells. On the other hand, the nonaxisymmetric magnetic field causes the neoclassical radial transport in a tandem mirror. ${ }^{3-9}$ It is desirable to make an effort to stabilize interchange modes in a fully axisymmetric mirror cell. The magnetic divertor with nonparaxial mirror magnetic field is an axisymmetric mirror that is able to stabilize the interchange modes. Ryutov and Stupakov ${ }^{10}$ suggested that the nonparaxial magnetic field could suppress the interchange modes by the rapid increase of the specific volume in an axisymmetric mirror. The nonparaxial axisymmetric magnetic field can be created by superimposing the field of two point coils and a uniform field, which produces two magnetic null points. Ryutov and Stupakov find that the lowest largescale modes can be stabilized in the magnetic configuration, and the only perturbations which remained unstable were those localized at the plasma boundary.

Subsequent theoretical work of stabilizing interchange modes by magnetic divertor was done by Lane et al. ${ }^{11}$ Instead of the magnetohydrodynamic equation, they used the kinetic equation with a paraxial approximation, where the characteristic length of the cross-field pressure gradient is assumed to be much less than the radius of magnetic-field line curvatures. Lane et al. took into account the magneticfield line curvature as an effective centrifugal force in the analysis. Finite Larmor radius (FLR) effects in paraxial plasmas can stabilize all flute-like interchange modes except the rigid $m=1$ mode. The divertor-like separatrix with circular magnetic field null plays the roll of a short circuit at the plasma edge; it can stabilize the rigid $m=1$ mode due to the constancy of the plasma potential at the separatrix.

\footnotetext{
${ }^{a)}$ Electronic mail: katanuma@prc.tsukuba.ac.jp

${ }^{b)}$ Electronic mail: past@nfi.kiae.ru
}

Pastukhov and Sokolov ${ }^{12}$ modified Lane's theory by adding nonparaxial magnetic-field lines and made the effect of field line curvature and ion FLR around the magnetic null in a magnetic divertor region explicit. As a result, they found that plasma pressure localized near the axis is always unstable to the interchange modes, and that the FLR effects become important only if the ion Larmor radius $\rho_{i}$ is comparable with the plasma radius $a$, i.e., $\rho_{i} / a>0.3$. The main stabilizing effects in the nonparaxial magnetic field with unfavorable curvature is found from the plasma compressibility.

The design of a magnetic divertor is a recent issue in the attempt to improve the radial confinement of the GAMMA10 tandem mirror ${ }^{14}$ with the developments of the theoretical study. ${ }^{15,16}$ Experiments on the magnetic divertor were carried out at the TARA tandem mirror ${ }^{17}$ in which complete divertor stabilization of the central cell plasma was not achieved in these experiments because the plasma pressure was localized near the axis, but some indirect evidence of stabilizing influence was observed. In contrast, the HIEI tandem mirror ${ }^{18}$ has been able to stabilize the interchange modes with wider plasma density radial profiles.

The GAMMA10 tandem mirror is the largest tandem mirror in operation in the world. It contains nonaxisymmetric quadrupole mirror cells for its magnetohydrodynamic (MHD) stability. ${ }^{19}$ High plasma pressure is required in the nonaxisymmetric mirror cells to stabilize the plasma in the central cell of GAMMA10 (Refs. 20 and 21). In order to reduce the load on the nonaxisymmetric mirror cells, there is a plan to install a magnetic divertor in the central cell of GAMMA10 without changing the present coil configuration. ${ }^{22}$

In this work we generalize the work of Pastukhov and Sokolov $^{12}$ to take into account the ion temperature radial dependence and the ion temperature anisotropy. 


\section{FLUTE-MODE EQUATION}

We consider an axisymmetric and nonparaxial plasma with anisotropic ion temperature. We introduce the coordinates $(\psi, \theta, \chi)$ to describe the basic equation, where the magnetic field expressed in terms of these coordinates is $\boldsymbol{B}=\nabla \chi=\nabla \psi \times \nabla \theta$ using the assumption of a low $\beta$ plasma. Here $\beta$ is the ratio of the plasma pressure to the magnetic pressure, $2 \pi \psi$ is the magnetic flux inside the surface of $\psi=$ const, $\theta$ is the azimuthal angle, and $\chi$ is the coordinate along the magnetic-field line. For a low $\beta$ plasma it is well known that the most dangerous modes are electrostatic flutelike perturbations such as $\phi_{1}=\phi_{1}(\psi) \exp \{-\mathrm{i} \omega t+\mathrm{i} m \theta\}$, where the mode amplitude $\phi_{1}(\psi)$ is uniform along magnetic-field lines and $m$ is a mode number in the azimuthal direction. The flute-like mode equation for $\phi_{1}$ is obtained by the charge neutrality condition within the infinitely thin magnetic flux tube.

As shown later in this section, the ion distribution function is assumed to be non-Maxwellian so that there exists an ion density variation along magnetic-field lines in steady state. The ion density gradient leads to the zeroth-order electrostatic potential $\phi_{0}(\chi)$ that varies along magnetic-field lines and is determined by the charge neutrality condition. The effect of $\phi_{0}(\chi)$ on the perturbed charge neutrality condition is investigated in Appendix A. In the following analysis, $\phi_{0}(\chi)$ is assumed to have a negligibly small effect on the ion dynamics under the assumption that electron temperature $T_{e}$ is sufficiently small in comparison to the perpendicular component of ion temperature $T_{i \perp}$.

\section{A. Ion motion}

In this section we derive the ion perturbed density $n_{i 1}$ due to the flute-like perturbation. Ion motion in an axisymmetric mirror field is described by the Lagrangian,

$$
\mathcal{L}=\frac{1}{2} M_{i} \boldsymbol{v}^{2}+\frac{e \boldsymbol{v}}{c} \cdot \boldsymbol{A}-e \phi_{1} .
$$

Here we use standard notations throughout the paper, i.e., $M_{i}$, ion mass; $e$, unit charge; $c$, the light speed; $\boldsymbol{v}$, the ion velocity; and $\phi_{1}$ represents the perturbed electrostatic potential, respectively. Recalling that $\boldsymbol{B}=\nabla \psi \times \nabla \theta=\nabla \times(\psi \nabla \theta)$, so that the vector potential $\boldsymbol{A}=\psi \nabla \theta$, the Lagrangian $\mathcal{L}$ is represented in the coordinates $(\psi, \theta, \chi)$ by

$$
\mathcal{L}=\frac{1}{2} M_{i}\left[\left(\frac{\dot{\psi}}{r B}\right)^{2}+(r \dot{\theta})^{2}+\left(\frac{\dot{\chi}}{B}\right)^{2}\right]+\frac{e}{c} \psi \dot{\theta}-e \phi_{1} .
$$

Here $\boldsymbol{B}$ is magnetic field and the symbol dot represents the time derivative. Now we introduce new variables $u_{i}, q_{i}$ defined by

$$
u_{i} \equiv \frac{e \psi}{M_{i} c}, \quad q_{i} \equiv \frac{e \chi}{M_{i} c} .
$$

In the axisymmetric system the Lagrangian $\mathcal{L}$ does not include the $\theta$ variable, so that the angular momentum $p_{\theta}$ is conserved,

$$
p_{\theta}=\frac{\partial \mathcal{L}}{\partial \dot{\theta}}=M_{i} r^{2} \dot{\theta}+M_{i} u_{i} \equiv M_{i} u_{0}=\text { const. }
$$

Here $u_{0}$ is the first integral corresponding to the angular velocity of ion guiding center position.

In order to include the $\psi$ dependence of the ion density and temperature, the ion distribution function is assumed to be

$$
\begin{aligned}
f_{i 0}\left(p_{\theta}, v_{\|}, v_{\perp}\right)= & n_{i}\left(p_{\theta}\right)\left(\frac{M_{i}}{2 \pi T_{i \perp}\left(p_{\theta}\right)}\right)\left(\frac{M_{i}}{2 \pi T_{i \|}\left(p_{\theta}\right)}\right)^{1 / 2} \\
& \times \exp \left\{-\frac{M_{i} v_{\|}^{2}}{2 T_{i \|}\left(p_{\theta}\right)}-\frac{M_{i} v_{\perp}^{2}}{2 T_{i \perp}\left(p_{\theta}\right)}\right\} .
\end{aligned}
$$

Here $v_{\|}\left(v_{\perp}\right)$ are the local ion velocity components parallel (perpendicular) to the magnetic-field line. Ion local velocities $v_{\|}$and $v_{\perp}$ are a function of $\chi$ through $v_{\|}=v_{\|}(\epsilon, \mu)$, $v_{\perp}=v_{\perp}(\epsilon, \mu)$, where ion energy $\epsilon$ and magnetic moment $\mu$ are conserved along the ion orbit. The equilibrium electrostatic potential $\phi_{0}$ resulting from the ion and electron charge neutrality condition is of the order of $e \phi_{0} \sim T_{e}$, so that $\phi_{0}$ can be neglected in the ion distribution function on the assumption of $e \phi_{0} \ll T_{i}$ in the case of $T_{e}<T_{i}$.

In order to calculate ion density in configuration space we expand the ion distribution function with respect to $r v_{\theta}$ assuming that $\rho_{i} \nabla=r v_{\theta} \partial / \partial u_{i} \ll 1$, where $v_{\theta}=r \dot{\theta}$ and $\rho_{i}$ is ion Larmor radius defined by $\rho_{i}=v_{\theta} / \omega_{c i}$ with $\omega_{c i}=e B / M_{i} c$. We then find

$$
f_{i 0}\left(r v_{\theta}+u_{i}\right) \simeq f_{i 0}\left(u_{i}\right)+r v_{\theta} \frac{\partial f_{i 0}\left(u_{i}\right)}{\partial u_{i}}+\frac{\left(r v_{\theta}\right)^{2}}{2} \frac{\partial^{2} f_{i 0}\left(u_{i}\right)}{\partial u_{i}^{2}} .
$$

Ion density $n_{i 0}$ of the order $\left(\rho_{i} \nabla\right)^{2}$ is obtained by integrating Eq. (6) with respect to local velocity space,

$$
\begin{aligned}
n_{i 0} & =\int_{0}^{\infty} v_{\perp} \mathrm{d} v_{\perp} \int_{-\infty}^{\infty} \mathrm{d} v_{\|} f_{i 0} \\
& =n_{i}+r^{2}\left(\frac{T_{i \perp}}{2 M_{i}} n_{i}^{\prime \prime}+\frac{T_{i \perp}^{\prime}}{M_{i}} n_{i}^{\prime}+\frac{T_{i \perp}^{\prime \prime}}{2 M_{i}} n_{i}\right),
\end{aligned}
$$

where $n_{i}=n_{i}\left(p_{\theta} \rightarrow \psi\right)$. Here the use of primes as superscripts means the derivative with respect to $u_{i}$. The charge neutrality condition in the equilibrium state is $n_{i 0}=n_{e 0}$.

\section{B. Perturbed ion distribution function}

The perturbed ion distribution function $f_{i 1}$ is obtained from the linearized Vlasov equation as

$$
\frac{\partial f_{i 1}}{\partial t}+\boldsymbol{v} \cdot \nabla f_{i 1}+\frac{e}{M_{i} c} \boldsymbol{v} \times \boldsymbol{B}_{0} \cdot \nabla_{v} f_{i 1}=\frac{e}{M_{i}} \nabla \phi_{1} \cdot \nabla_{v} f_{i 0} .
$$

Here the subscripts 0 and 1 represent equilibrium and perturbed quantities, respectively. Integrating Eq. (8) along the zeroth ion orbit, ${ }^{11}$ 


$$
\begin{aligned}
f_{i 1}= & \int_{-\infty}^{t} \frac{e}{M_{i}} \nabla^{\prime} \phi_{1} \cdot \nabla_{v^{\prime}} f_{i 0} \mathrm{~d} t^{\prime} \\
= & \frac{e}{M_{i}} \int_{-\infty}^{t}\left[M_{i}\left(\frac{\mathrm{d} \phi_{1}}{\mathrm{~d} t^{\prime}}-\frac{\partial \phi_{1}}{\partial t^{\prime}}\right) \frac{\partial f_{i 0}}{\partial \epsilon}+\frac{M_{i} c}{e} \frac{\partial \phi_{1}}{\partial \theta^{\prime}} \frac{\partial f_{i 0}}{\partial \psi}\right] \mathrm{d} t^{\prime} \\
= & e \phi_{1} \frac{\partial f_{i 0}}{\partial \epsilon}+\mathrm{i} e\left(\omega \frac{\partial f_{i 0}}{\partial \epsilon}+\frac{m c}{e} \frac{\partial f_{i 0}}{\partial \psi}\right) \int_{-\infty}^{t} \phi_{1}\left(\psi^{\prime}\right) \\
& \times \exp \left\{-\mathrm{i} \omega t^{\prime}+\mathrm{i} m \theta^{\prime}\right\} \mathrm{d} t^{\prime} .
\end{aligned}
$$

The ion motion consists of Larmor motion and guiding center drift motion resulting from magnetic-field line curvature,

$$
u_{i}-u_{0}=Z+W \cos y, \quad \dot{u}_{i}=\dot{Z}-\omega_{c i} W \sin y .
$$

Here $Z$ is the slow change in $u_{i}$, i.e., the angular velocity of drift motion, and $W \cos y$ corresponds to the fast oscillation around the mean magnetic flux coordinate $\psi$, i.e., the angular velocity of Larmor motion. The variables $Z, W, y$ are taken as new functions defined by Eq. (10). After the ion motion of $u_{i}$ is obtained from Lagrangian (2) and is taken into account of the order of $\left(\rho_{i} \nabla\right)^{2}$, we have, following Ref. 12,

$$
\begin{aligned}
& \dot{y}=\omega_{c i}\left(u_{0}, q_{i}\right), \\
& Z=\frac{W^{2}}{r} \frac{\partial r}{\partial u_{i}}-\frac{1}{\omega_{c i}}\left(\frac{W^{2}}{2}+\frac{\dot{q}_{i}^{2} r^{2}}{\omega_{c i}^{2}}\right) \frac{\partial \omega_{c i}}{\partial u_{i}}, \frac{\mathrm{d}}{\mathrm{d} t}\left(\frac{W^{2}}{r^{2} \omega_{c i}}\right)=0 .
\end{aligned}
$$

In order to carry out the integral in Eq. (9), we assume that the characteristic frequency of the interchange mode under consideration is much slower than the ion Larmor frequency,

$$
\begin{aligned}
\int_{-\infty}^{t} \phi_{1}\left(u_{i}^{\prime}\right) \exp \left\{-\mathrm{i} \omega t^{\prime}+\mathrm{i} m \theta^{\prime}\right\} \mathrm{d} t^{\prime} & =\int_{-\infty}^{t} \phi_{1}\left(u_{i}^{\prime}\right) \exp \left\{-\mathrm{i} \omega t^{\prime}+\mathrm{i} m\langle\dot{\theta}\rangle\left(t^{\prime}-t\right)+\mathrm{i} m \theta\right\} \times \exp \left\{\mathrm{i} m \int_{t}^{t^{\prime}}(\dot{\theta}-\langle\dot{\theta}\rangle) \mathrm{d} t^{\prime \prime}\right\} \mathrm{d} t^{\prime} \\
= & \sum_{n=-\infty}^{\infty} \exp \left\{-\mathrm{i} \omega t^{\prime}+\mathrm{i} m\langle\dot{\theta}\rangle\left(t^{\prime}-t\right)+\mathrm{i} m \theta\right\} \int_{t_{n-1}}^{t_{n}} \phi_{1}\left(u_{i}^{\prime}\right) \exp \left\{\mathrm{i} m \int_{t}^{t^{\prime}}(\dot{\theta}-\langle\dot{\theta}\rangle) \mathrm{d} t^{\prime \prime}\right\} \mathrm{d} t^{\prime} \\
= & \sum_{n=-\infty}^{\infty} \frac{2 \pi}{\omega_{c i}}\left\langle\phi_{1}\right\rangle \exp \left\{-\mathrm{i} \omega t^{\prime}+\mathrm{i} m\langle\dot{\theta}\rangle\left(t^{\prime}-t\right)+\mathrm{i} m \theta\right\}=\int_{-\infty}^{t}\left\langle\phi_{1}\right\rangle \exp \left\{-\mathrm{i} \omega t^{\prime}+\mathrm{i} m\langle\dot{\theta}\rangle\left(t^{\prime}-t\right)\right. \\
& +\mathrm{i} m \theta\} \mathrm{d} t^{\prime},
\end{aligned}
$$

where the average quantity $\langle\dot{\theta}\rangle$ is defined as

$$
\langle\dot{\theta}\rangle \equiv \frac{\omega_{c i}}{2 \pi} \int_{0}^{2 \pi / \omega_{c i}} \dot{\theta} \mathrm{d} t^{\prime}
$$

and the average quantity $\left\langle\phi_{1}\right\rangle$ is defined as

$$
\left\langle\phi_{1}\right\rangle \equiv \frac{\omega_{c i}}{2 \pi} \int_{0}^{2 \pi / \omega_{c i}} \phi_{1}\left(u_{i}^{\prime}\right) \exp \left\{\mathrm{i} m \int_{t}^{t^{\prime}}(\dot{\theta}-\langle\dot{\theta}\rangle) \mathrm{d} t^{\prime \prime}\right\} \mathrm{d} t^{\prime} .
$$

Here the time interval is $t_{n}-t_{n-1}=2 \pi / \omega_{c i}$ and we make use of the high-frequency Chew-Goldberger-Low (CGL) approximation ${ }^{13}$ in Eq. (12). The function of $u_{i}$ is expanded by the ion Larmor radius around the guiding center for further calculation of Eqs. (12)-(14),

$$
\begin{aligned}
\phi_{1}\left(u_{i}\right)= & \phi_{1}\left(u_{0}\right)+\left(u_{i}-u_{0}\right) \frac{\partial \phi_{1}\left(u_{0}\right)}{\partial u_{0}} \\
+ & \frac{1}{2}\left(u_{i}-u_{0}\right)^{2} \frac{\partial^{2} \phi_{1}\left(u_{0}\right)}{\partial u_{0}^{2}}, \\
\exp \left\{\mathrm{i} m \int_{t}^{t^{\prime}}(\dot{\theta}-\langle\dot{\theta}\rangle) \mathrm{d} t^{\prime \prime}\right\} & =1+\mathrm{i} m \int_{t}^{t^{\prime}}(\dot{\theta}-\langle\dot{\theta}\rangle) \mathrm{d} t^{\prime \prime} \\
& -\frac{1}{2} m^{2}\left[\int_{t}^{t^{\prime}}(\dot{\theta}-\langle\dot{\theta}\rangle) \mathrm{d} t^{\prime \prime}\right]^{2} .
\end{aligned}
$$

Noting that $r\langle\dot{\theta}\rangle$ is equal to the ion drift velocity $v_{d \theta}$, where $\quad v_{d \theta}=-\left[c M_{i}\left(v_{\|}^{2}+\frac{1}{2} v_{\perp}^{2}\right) \nabla B \times \boldsymbol{B}\right] /\left.e B^{3}\right|_{\theta}=\left(v_{\|}^{2}\right.$ $\left.+\frac{1}{2} v_{\perp}^{2}\right)_{r}\left[\partial \ln B / \partial u_{i}\right],\langle\dot{\theta}\rangle$ is described as

$$
\langle\dot{\theta}\rangle=\left(v_{\|}^{2}+\frac{1}{2} v_{\perp}^{2}\right) \frac{\partial \ln B}{\partial u_{i}} .
$$




$$
\begin{aligned}
\dot{\theta}-\langle\dot{\theta}\rangle= & \frac{W^{2}}{r^{3}} \frac{\partial r}{\partial u_{i}} \cos \{2 y\}-\frac{W}{r^{2}} \cos \{y\}\left[1-\frac{4 W^{2}}{r^{2}}\left(\frac{\partial r}{\partial u_{i}}\right)^{2}\right. \\
& \left.+4 r\langle\dot{\theta}\rangle \frac{\partial r}{\partial u_{i}}\right] .
\end{aligned}
$$

Because the variable $y$ corresponds to the ion gyrophase, the integration on the argument of exponential in Eq. (14) can be carried out as

$$
\begin{aligned}
\int_{t}^{t^{\prime}}(\dot{\theta}-\langle\dot{\theta}\rangle) \mathrm{d} t^{\prime \prime}= & \frac{W^{2}}{r^{3} \omega_{c i}} \frac{\partial r}{\partial u_{i}} \sin \left\{\omega_{c i} \tau\right\} \cos \left\{\omega_{c i} \tau+2 \xi\right\} \\
& -\frac{2 W}{r^{2} \omega_{c i}}\left[1-\frac{4 W^{2}}{r^{2}}\left(\frac{\partial r}{\partial u_{i}}\right)^{2}\right. \\
& \left.+4 r\langle\dot{\theta}\rangle \frac{\partial r}{\partial u_{i}}\right] \sin \left\{\frac{\omega_{c i} \tau}{2}\right\} \cos \left\{\frac{\omega_{c i} \tau}{2}+\xi\right\},
\end{aligned}
$$

by setting $y=\omega_{c i} \tau+\xi$, where $\xi$ is an initial phase. Inserting Eqs. (15) and (18) into Eq. (14) and carrying out the integration on $t^{\prime}$ over one gyroperiod, we obtain

$$
\begin{aligned}
\left\langle\phi_{1}\right\rangle= & \phi_{1}\left(u_{0}\right)\left[1+i m\left\{-\frac{v_{\perp}^{2}}{2 r \omega_{c i}} \frac{\partial r}{\partial u_{i}} \sin (2 \xi)+\frac{v_{\perp}}{r \omega_{c i}} \sin \xi\right\}\right. \\
& \left.-\frac{1}{4} m^{2}\left(\frac{v_{\perp}}{r \omega_{c i}}\right)^{2} \sin ^{2} \xi\right]+\frac{\partial \phi_{1}\left(u_{0}\right)}{\partial u_{i}}\left\{r v_{\perp}-2 \frac{\partial r}{\partial u_{i}}\right. \\
& \left.-r^{2}\left(\frac{1}{2} v_{\perp}^{2}+v_{\|}^{2} \frac{\partial \ln B}{\partial u_{i}}\right)\right\}+\frac{1}{4} \frac{\partial^{2} \phi_{1}\left(u_{0}\right)}{\partial u_{i}^{2}} r^{2} v_{\perp}^{2}
\end{aligned}
$$

Up until now, we have calculated the variable $\left\langle\phi_{1}\right\rangle$ as a function of $u_{0}$, where $u_{0}$ corresponds to the ion guiding center radial position as defined by Eqs. (3) and (4). However, the variable $\left\langle\phi_{1}\right\rangle$ is a function of radial position $u_{i}$, and therefore it is necessary to carry out the integration of Eq. (12). Because the argument of the variable $\phi_{1}$ in the right-hand side of Eq. (19) is $u_{0}$, the perturbed electrostatic potential $\phi_{1}\left(u_{i}\right)$ can be determined by using the Taylor expansion in Eq. (15),

$$
\phi_{1}\left(u_{0}\right)=\phi_{1}\left(u_{i}\right)+\left(u_{0}-u_{i}\right) \frac{\partial \phi_{1}\left(u_{i}\right)}{\partial u_{i}}+\frac{1}{2}\left(u_{0}-u_{i}\right)^{2} \frac{\partial^{2} \phi_{1}\left(u_{i}\right)}{\partial u_{i}^{2}} .
$$

The result of the integration in Eq. (12) is

$$
\begin{aligned}
& \int_{-\infty}^{t}\left\langle\phi_{1}\right\rangle \exp \left\{-\mathrm{i} \omega t^{\prime}+\mathrm{i} m\langle\dot{\theta}\rangle\left(t^{\prime}-t\right)+\mathrm{i} m \theta\right\} \mathrm{d} t^{\prime} \\
& =\frac{\mathrm{i}}{\omega-m\langle\dot{\theta}\rangle}\left\langle\phi_{1}\right\rangle \exp \{-\mathrm{i} \omega t+\mathrm{i} m \theta\} \\
& \simeq \frac{\mathrm{i}}{\omega}\left[1+\frac{m}{\omega}\langle\dot{\theta}\rangle+\left(\frac{m}{\omega}\langle\dot{\theta}\rangle\right)^{2}\right]\left\langle\phi_{1}\right\rangle \exp \{-\mathrm{i} \omega t+\mathrm{i} m \theta\},
\end{aligned}
$$

on the assumption of $\omega \gg m\langle\dot{\theta}\rangle$. Here $\langle\dot{\theta}\rangle$ and $\left\langle\phi_{1}\right\rangle$ are given by Eqs. (16), (19), and (20). Finally, the perturbed distribution function is explicitly determined by Eq. (9) and the perturbed ion density $n_{i 1}$ is determined by integrating $f_{i 1}$ in velocity space,

$$
\begin{aligned}
n_{i 1}= & \int_{0}^{\infty} \mathrm{d} v_{\perp} \int_{-\infty}^{\infty} \mathrm{d} v_{\|} \int_{0}^{2 \pi} \mathrm{d} \xi f_{i 1} \\
= & \nabla \cdot\left[\frac{e}{M_{i} \omega_{c i}^{2}}\left(n_{i}-\frac{m T_{i \perp}}{M_{i} \omega} n_{i}^{\prime}-\frac{m n_{i}}{M_{i} \omega} T_{i \perp}^{\prime}\right) \nabla \phi_{1}\right] \\
& +\phi_{1}^{\prime}\left[n_{i}^{\prime} \frac{e m r^{2} B^{\prime}}{M_{i}^{2} B \omega}\left(T_{i \|}-T_{i \perp}\right)+n_{i} \frac{e r^{2} B^{\prime}}{M_{i} B}\left(1-\frac{T_{i \|}}{T_{i \perp}}+\frac{m T_{i \|}^{\prime}}{M_{i} \omega}-\frac{m T_{i \perp}^{\prime}}{M_{i} \omega}\right)\right] \\
& +\phi_{1}\left[-n_{i}^{\prime \prime \prime} \frac{e m r^{2} T_{i \perp}}{2 M_{i}^{2} \omega}-n_{i}^{\prime \prime} \frac{3 e m r^{2} T_{i \perp}^{\prime}}{2 M_{i}^{2} \omega}+n_{i}^{\prime}\left(-\frac{e m}{M_{i} \omega}-\frac{e m^{2} B^{\prime}\left(T_{i \|}+T_{i \perp}\right)}{B M_{i}^{2} \omega^{2}}-\frac{e m^{3} B^{\prime 2}\left(3 T_{i \|}^{2}+2 T_{i \|} T_{i \perp}+2 T_{i \perp}^{2}\right)}{B^{2} M_{i}^{3} \omega^{3}}-\frac{3 e m r^{2} T_{i \perp}^{\prime \prime}}{2 M_{i}^{2} \omega}\right)\right. \\
& +n_{i}\left(\frac{e m B^{\prime}}{B M_{i} \omega}\left(1+\frac{T_{i \|}}{T_{i \perp}}\right)+\frac{e m^{2} B^{\prime 2}}{B^{2} M_{i}^{2} \omega^{2}}\left(2 T_{i \|}+\frac{3 T_{i \|}^{2}}{T_{i \perp}}+2 T_{i \perp}\right)-\frac{e m^{2} B^{\prime}\left(T_{i \|}^{\prime}+T_{i \perp}^{\prime}\right)}{B M_{i}^{2} \omega^{2}}\right. \\
& \left.\left.-\frac{e m^{3} B^{\prime 2}\left(6 T_{i \|} T_{i \|}^{\prime}+2 T_{i \perp} T_{i \|}^{\prime}+2 T_{i \|} T_{i \perp}^{\prime}+4 T_{i \perp} T_{i \perp}^{\prime}\right)}{B^{2} M_{i}^{3} \omega^{3}}-\frac{e m r^{2} T_{i \perp}^{\prime \prime \prime}}{2 M_{i}^{2} \omega}\right)\right] .
\end{aligned}
$$


Here all variables in Eq. (22) are a function of $u_{i}$ and $\chi$ and the prime ' means the derivative with respect to $u_{i}$.

\section{Perturbed electron density}

Equation (22) can be applied to obtain the electron density perturbation $n_{e 1}$ by replacing $\left(n_{i 1}, T_{i \perp}, T_{i \|}, e\right)$ $\rightarrow\left(n_{e 1}, T_{e \perp}, T_{e \|},-e\right)$. It is assumed that the electron Larmor radius is negligibly small (i.e., $\rho_{e} \nabla \simeq 0$ ) and electron temperature is isotropic, $T_{e \perp}=T_{e \|}$, and very low, i.e., $T_{e \|}=T_{e \perp}$ $\simeq 0$. Recall that $1 / M_{e} \partial / \partial u_{e}=-1 / M_{i} \partial / \partial u_{i}$; the perturbed electron density is found to be

$$
n_{e 1}=\phi_{1}\left(2 n_{e} \frac{e m B^{\prime}}{B M_{i} \omega}-n_{e}^{\prime} \frac{e m}{M_{i} \omega}\right) .
$$

Using charge neutrality, the equilibrium electron density $n_{e 0}=n_{e}$ is replaced with $n_{i 0}$ in Eq. (7),

$$
n_{e}=n_{i}+r^{2}\left(\frac{T_{i \perp}}{2 M_{i}} n_{i}^{\prime \prime}+\frac{T_{i \perp}^{\prime}}{M_{i}} n_{i}^{\prime}+\frac{T_{i \perp}^{\prime \prime}}{2 M_{i}} n_{i}\right) .
$$

Therefore, the perturbed electron density $n_{e 1}$ is obtained as

$$
\begin{aligned}
n_{e 1}= & \phi_{1}\left[-n_{i}^{\prime \prime \prime} \frac{e m r^{2} T_{i \perp}}{2 M_{i}^{2} \omega}+n_{i}^{\prime \prime}\left(\frac{e m r^{2} T_{i \perp} B^{\prime}}{M_{i}^{2} \omega B}-\frac{3 e m r^{2} T_{i \perp}^{\prime}}{2 M_{i}^{2} \omega}\right)\right. \\
& +n_{i}^{\prime}\left(\frac{2 e m r^{2} T_{i \perp}^{\prime} B^{\prime}}{M_{i}^{2} \omega B}-\frac{3 e m T_{i \perp}^{\prime \prime} r^{2}}{2 M_{i}^{2} \omega}-\frac{e m}{M_{i} \omega}\right) \\
& \left.+n_{i}\left(\frac{2 e m B^{\prime}}{M_{i} \omega B}+\frac{e m r^{2} T_{i \perp}^{\prime \prime} B^{\prime}}{M_{i}^{2} \omega B}-\frac{e m r^{2} T_{i \perp}^{\prime \prime \prime}}{2 M_{i}^{2} \omega}\right)\right]
\end{aligned}
$$

\section{Local charge density}

Using Eqs. (22) and (23) and the relation $\partial / \partial u_{i}$ $=\left(M_{i} c / e \partial\right) / \partial \psi$, we obtain the local charge density as

$$
\begin{aligned}
\frac{e n_{i 1}-e n_{e 1}}{e^{2}}= & B^{2} \frac{\partial}{\partial \psi}\left[\frac{r^{2}}{B^{2}} n_{i}\left(1-\frac{m c}{e \omega} \frac{1}{n_{i}} \frac{\partial\left(n_{i} T_{i \perp}\right)}{\partial \psi}\right) \frac{\partial \phi_{1}}{\partial \psi}\right]-\phi_{1} \frac{m^{2}}{r^{2} B^{2}} n_{i}\left(1-\frac{m c}{e \omega} \frac{1}{n_{i}} \frac{\partial\left(n_{i} T_{i \perp}\right)}{\partial \psi}\right) \\
& +\frac{\partial \phi_{1}}{\partial \psi}\left[-\frac{m c r^{2}}{e \omega} \frac{\partial(\ln B)}{\partial \psi} \frac{\partial\left\{n_{i}\left(T_{i \perp}-T_{i \|}\right)\right\}}{\partial \psi}+r^{2} \frac{\partial(\ln B)}{\partial \psi} n_{i}\left(1-\frac{T_{i \|}}{T_{i \perp}}\right)\right]+\phi_{1}\left[-\frac{m c}{2 e \omega}\left\{2 r^{2} \frac{\partial(\ln B)}{\partial \psi}-\frac{\partial\left(r^{2}\right)}{\partial \psi}\right\} \frac{\partial^{2}\left(n_{i} T_{i \perp}\right)}{\partial \psi^{2}}\right. \\
& -\frac{m^{2}}{M_{i} \omega^{2}} \frac{\partial(\ln B)}{\partial \psi} \frac{\partial\left\{n_{i}\left(T_{i \|}+T_{i \perp}\right)\right\}}{\partial \psi}+\frac{m e}{M_{i} \omega c} \frac{\partial(\ln B)}{\partial \psi} n_{i}\left(\frac{T_{i \|}}{T_{i \perp}}-1\right)+\frac{m^{2}}{M_{i} \omega^{2}}\left(\frac{\partial(\ln B)}{\partial \psi}\right)^{2} n_{i}\left(2 T_{i \|}+3 \frac{T_{i \|}^{2}}{T_{i \perp}}+2 T_{i \perp}\right) \\
& \left.-\frac{m^{3} c}{M_{i} e \omega^{3}}\left(\frac{\partial(\ln B)}{\partial \psi}\right)^{2} n_{i}\left(6 T_{i \|} T_{i \|}^{\prime}+2 T_{i \perp} T_{i \|}^{\prime}+2 T_{\|} T_{i \perp}^{\prime}+4 T_{i \perp} T_{i \perp}^{\prime}\right)\right]
\end{aligned}
$$

Here all variables in Eq. (24) are a function of $\psi$ and $\chi$, and the prime means the derivative with respect to $\psi$.

The ion distribution function $f_{i 0}\left(u_{i}\right)$, with $T_{i \perp} \neq T_{i \|}$ in $(\psi, \chi)$ space, is given by the first term in the right-hand side of Eq. (6) and the distribution function $f_{i 0}$, at $(\psi, \chi)$, is written as

$$
\begin{aligned}
f_{i 0}(\psi, \chi, \epsilon, \mu)= & n_{i}(\psi, \chi)\left(\frac{M_{i}}{2 \pi T_{i \perp}(\psi, \chi)}\right)\left(\frac{M_{i}}{2 \pi T_{i \|}(\psi, \chi)}\right)^{1 / 2} \\
& \times \exp \left\{-\frac{M_{i} v_{\|}^{2}}{2 T_{i \|}(\psi, \chi)}-\frac{M_{i} v_{\perp}^{2}}{2 T_{i \perp}(\psi, \chi)}\right\},
\end{aligned}
$$

where $v_{\|}, v_{\perp}$ are the local velocities at $(\psi, \chi)$, and $T_{i \perp}(\psi, \chi)$, $T_{i \|}(\psi, \chi), n_{i}(\psi, \chi)$ are related to $T_{i \perp c c}, T_{i \|_{c c}}, n_{i c c}$, such as

$$
\begin{aligned}
& T_{i \|}(\psi, \chi)=T_{i \| c c}(\psi), \\
& \frac{1}{T_{i \perp}(\psi, \chi)}=\frac{1}{T_{i \| c c}(\psi)}-\frac{B_{c c}(\psi)}{B(\psi, \chi) T_{i \| c c}(\psi)}+\frac{B_{c c}(\psi)}{B(\psi, \chi) T_{i \perp c c}(\psi)},
\end{aligned}
$$

$$
\begin{aligned}
n_{i}(\psi, \chi)= & \frac{n_{i c c}(\psi)}{T_{i \perp c c}(\psi)}\left(\frac{1}{T_{i \|_{c c}}(\psi)}-\frac{B_{c c}(\psi)}{B(\psi, \chi) T_{i \| c c}(\psi)}\right. \\
& \left.+\frac{B_{c c}(\psi)}{B(\psi, \chi) T_{i \perp c c}(\psi)}\right)^{-1} .
\end{aligned}
$$

Here the subscript $c c$ means the quantity at the midplane of the divertor mirror cell, and we have used the conservation of energy $\epsilon=\frac{1}{2} M_{i} v_{\|}^{2}+\frac{1}{2} M_{i} v_{\perp}^{2}$ and magnetic moment $\mu=\frac{1}{2} M_{i} v_{\perp}^{2} / B$. Therefore, $T_{i \perp}, T_{i \|}, n_{i}$ in Eq. (24) depend on the magnetic field $B$ through Eq. (26). The derivation of Eq. (26) is given in Appendix B.

\section{E. Flute-mode equation}

The solvability condition, which is given by Eq. (A10) in Appendix A, is written as

$$
\int \frac{e n_{i 1}-e n_{e 1}}{e^{2} B^{2}} \mathrm{~d} \chi=0
$$

Finally, using Eqs. (24) and (27), the stability analysis equation is obtained as 


$$
\begin{aligned}
\frac{\partial}{\partial \psi}[ & \left.n_{i c c}\left(H_{1,1}+m \frac{\omega_{c c}^{\star}}{\omega} H_{1,2}-m \frac{c T_{i \perp c c}}{e \omega} H_{1,3}\right) \frac{\partial \phi_{1}}{\partial \psi}\right]-k_{m}^{2} n_{i c c}\left(H_{2,1}+m \frac{\omega_{c c}^{\star}}{\omega} H_{2,2}-m \frac{c T_{i \perp c c}}{e \omega} H_{2,3}\right) \phi_{1} \\
& +\frac{\partial \phi_{1}}{\partial \psi}\left[\frac{m c}{e \omega}\left(\frac{e n_{i c c} \omega_{c c}^{\star}}{c} H_{3,2}+\frac{\partial\left(n_{i c c} T_{i \| c c}\right)}{\partial \psi} H_{3,1}-n_{i c c} T_{i \perp c c} H_{3,3}+n_{i c c} T_{i \| c c} H_{3,4}\right)+\left(n_{i c c} H_{3,1}-\frac{n_{i c c} T_{i \| c c}}{T_{i \perp c c}} H_{3,5}\right)\right] \\
& +\phi_{1}\left[-\frac{m}{2}\left(H_{4,1} \frac{\partial}{\partial \psi}\left(\frac{n_{i c c} \omega_{c c}^{\star}}{\omega}\right)-H_{4,2} \frac{\partial}{\partial \psi}\left(\frac{c n_{i c c} T_{i \perp c c}}{e \omega}\right)+\frac{n_{i c c} \omega_{c c}^{\star}}{\omega} H_{4,2}-\frac{c n_{i c c} T_{i \perp c c}}{e \omega} H_{4,3}\right)-\frac{k_{m}^{2}}{M_{i} \omega^{2}}\left(\frac{\partial\left(n_{i c c} T_{i \| c c}\right)}{\partial \psi} H_{5,1}\right.\right. \\
& \left.-\frac{e n_{i c c} \omega_{c c}^{\star}}{c} H_{5,2}+n_{i c c} T_{i \| c c} H_{5,4}+n_{i c c} T_{i \perp c c} H_{5,3}\right)-\frac{m e}{M_{i} \omega c}\left(n_{i c c} H_{5,1}-\frac{n_{i c c} T_{i \| c c}}{T_{i \perp c c}} H_{5,5}\right) \\
& +\frac{k_{m}^{2}}{M_{i} \omega^{2}}\left(2 n_{i c c} T_{i \| c c} H_{6,1}+3 \frac{n_{i c c} T_{i \|}^{2}}{T_{i \perp c c}} H_{6,5}+2 n_{i c c} T_{i \perp c c} H_{6,2}\right)-\frac{k_{m}^{3} c}{M_{i} e \omega^{3}} n_{i c c} \\
& \left.\times\left(3 \frac{\partial T_{i \| c c}^{2}}{\partial \psi} H_{6,1}+2 \frac{\partial\left(T_{i \perp c c} T_{i \| l c c}\right)}{\partial \psi} H_{6,2}+2 \frac{\partial T_{i \perp c c}^{2}}{\partial \psi} H_{6,6}+T_{i \perp c c} T_{i \| c c} H_{6,3}+\frac{4}{3} T_{i \perp c c}^{2} H_{6,4}\right)\right]=0,
\end{aligned}
$$

where the coefficients $H_{i, j}$ are given in Appendix C. Here

$$
\begin{aligned}
& \omega_{c c}^{\star}=-\frac{c}{e n_{i c c}} \frac{\partial\left(n_{i c c} T_{i \perp c c}\right)}{\partial \psi}, \\
& h_{\text {local }}(\psi, \chi)=\left(\frac{T_{i \perp}(\psi, \chi)}{T_{i \|}(\psi, \chi)}-\frac{B_{c c}(\psi) T_{i \perp}(\psi, \chi)}{B(\psi, \chi) T_{i \|}(\psi, \chi)}+\frac{B_{c c}(\psi)}{B(\psi, \chi)}\right)^{-1},
\end{aligned}
$$

$n_{i c c}(\psi)$ is ion density in the equilibrium state, $T_{i \perp c c}$ and $T_{i \| c c}$ are perpendicular and parallel ion temperature at the midplane, respectively, and $\omega$ is the frequency of flute interchange mode.

In the special case that $T_{i \perp c c}=T_{i \| c c}=T_{i}$ and $T_{i}$ are independent of $\psi$, Eq. (28) reduces to Eq. (1) in Ref. 12. Comparison of Eq. (28) in this paper and Eq. (1) in Ref. 12 reveals that two new terms appear in this stability equation (28): ion temperature anisotropy and the radial dependence of ion temperatures. In particular it is clear that the term proportional to $\partial \phi_{1} / \partial \psi$ appears only if $T_{i \perp} \neq T_{i \|}$ in Eq. (28). And the last term in the right-hand side of Eq. (28) appears only if the temperature gradient exists.

Equation (28) is the second-order differential equation of $\phi_{1}$ with respect to $\psi$, so that the two boundary conditions are necessary to solve the equation. One boundary condition assures that $\phi_{1}$ has an asymptotic solution $\phi \sim \psi^{m / 2}$ near $\psi=0$ (Ref. 12).

Another boundary condition requires that $\phi_{1}=0$ at $\psi=\psi_{s}$, where $\psi_{s}$ is the coordinate of magnetic null, i.e., a magnetic-field line on the surface $\psi_{s}$ passes through the magnetic null point. This boundary condition is zero perturbed potential on the divertor field line. Electrons can stream both axially and azimuthally due to loss of adiabaticity near a magnetic null region. The azimuthal stream of electrons plays a role of short circuit of perturbed electrostatic potential $\phi_{1}$ on the annular magnetic flux line tube. Electrons can also move along a magnetic-field line of the divertor, which makes $\phi_{1}=0$ along the magnetic-field line. Therefore, the boundary condition of $\phi_{1}=0$ at $\psi=\psi_{s}$ is suitable in this case.
The behavior of $\phi_{1}$ around the magnetic-field line of the divertor was discussed by Lane et al. ${ }^{11}$ The above boundary condition is a rough model that needs to be verified and most likely changed by examining the kinetic response of particles near and on the open field line of the divertor.

Therefore Eq. (28) is a eigenequation with eigenvalue $\omega$, and then the eigenvalue $\omega$ can be determined by solving Eq. (28) with the above boundary condition of $\phi_{1}$. If the imaginary part of $\omega$ is negative, then the system is stable to the flute modes; while the system is unstable to the flute modes if the imaginary part of $\omega$ is positive.

\section{NUMERICAL CALCULATION OF EQ. (28)}

In this section the numerical results of Eq. (28) are presented. The functional forms of $T_{i \perp c c}(\psi)$ and $T_{i \| c c}(\psi)$ are given in advance. However, the coefficients of $\phi_{1}$ contain the differential of $B$ with respect to $\psi$ and an integration along a magnetic-field line. The direct calculation of the partial derivative of $B$ with respect to $\psi$ must be carried out on the constant $\chi$ plane, but the numerical calculation is difficult to perform within the required numerical accuracy. Therefore, we use the following relation of $\partial B / \partial \psi$ and $\partial^{2} B / \partial^{2} \psi$. In the vacuum magnetic field satisfies

$$
\nabla \times B \hat{e}_{\|}=\nabla B \times \hat{e}_{\|}+B \nabla \times \hat{e}_{\|}=0,
$$

so that

$$
\begin{aligned}
\nabla_{\perp} B & =B\left(\nabla \times \hat{e}_{\|}\right) \times \hat{e}_{\|}=B \hat{e}_{\|} \cdot \nabla \hat{e}_{\|} \\
& =B \frac{\partial}{\partial \ell}\left(\frac{\partial r(\psi, \ell)}{\partial \ell} \hat{e}_{r}+\frac{\partial z(\psi, \ell)}{\partial \ell} \hat{e}_{z}\right) .
\end{aligned}
$$

Here $(r, z)$ are the radial and axial coordinates of a magneticfield line $(\psi, \chi)$; the coordinate $\ell$ along the magnetic field line is defined as $B \nabla \ell \equiv \nabla \chi$, and $\hat{e}_{\|}$is the unit vector along a magnetic-field line. Noting that $\nabla_{\perp} B=\nabla \psi \partial B / \partial \psi$ and $\boldsymbol{B}=\nabla \psi \times \hat{e}_{\theta} / r$, we obtain the following relation: 

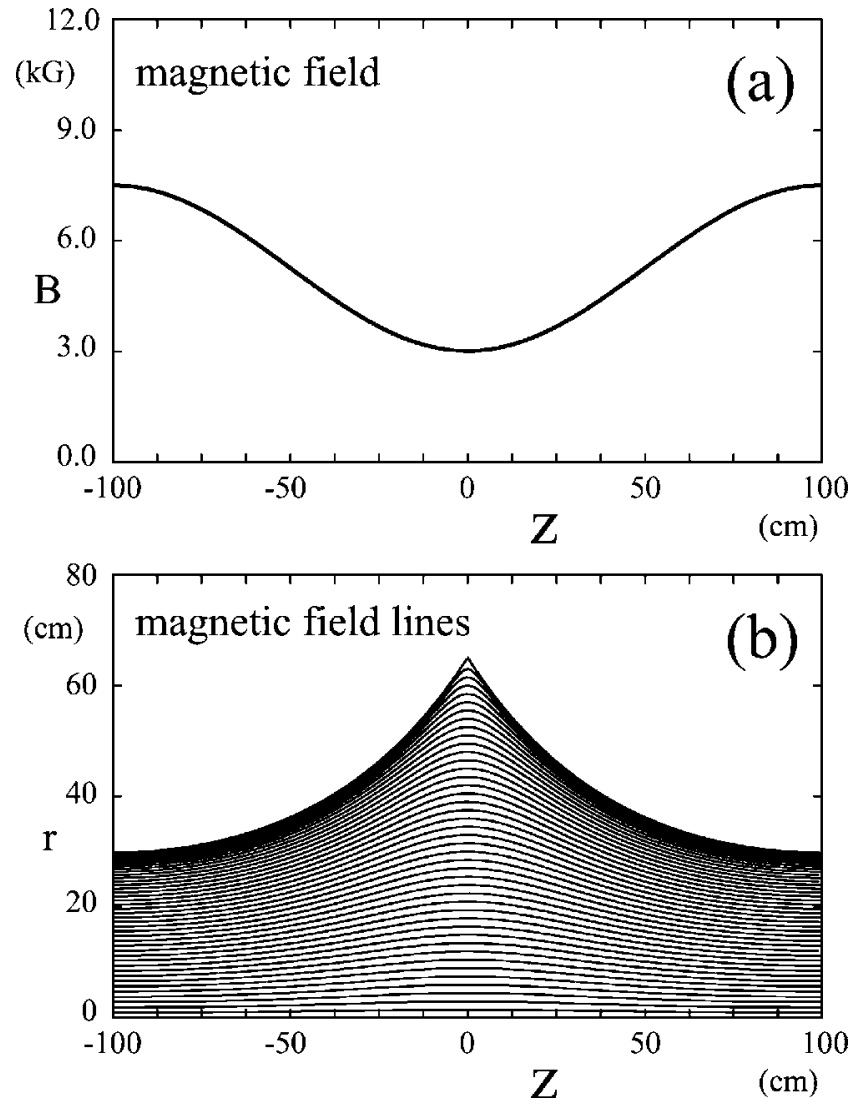

FIG. 1. The axial magnetic-field strength (a) and magnetic-field lines.

$$
\frac{\partial B}{\partial \psi}=\frac{B}{r B_{z}} \frac{\partial^{2} r}{\partial \ell^{2}}=-\frac{B}{r B_{r}} \frac{\partial^{2} z}{\partial \ell^{2}} .
$$

The formula for the second derivative, $\partial^{2} B / \partial \psi^{2}$, is found in Appendix 4 in Ref. 10. Although the expression in Ref. 10 is written in terms of the distance between the magnetic flux tube, the expression in terms of $\psi, \ell$ is rewritten easily as

$$
\begin{aligned}
\frac{\partial^{2} B}{\partial \psi^{2}}= & \frac{1}{B}\left(\frac{\partial B}{\partial \psi}\right)^{2}+\frac{2}{r^{2} B^{3}}\left(\frac{\partial B}{\partial \ell}\right)^{2}-\frac{2 B_{z}}{r^{2} B^{2}} \frac{\partial B}{\partial \psi}+\frac{2}{r^{2} B^{3}}\left(\frac{B_{r}}{r}\right)^{2} \\
& +\frac{2 B_{r}}{r^{3} B^{3}} \frac{\partial B}{\partial \ell}-\frac{1}{r^{2} B^{2}} \frac{\partial^{2} B}{\partial \ell^{2}}
\end{aligned}
$$

The following model is adopted as a divertor magneticfield configuration: ${ }^{12}$

$$
\begin{aligned}
& B_{z}=B_{0}\left(1-\frac{1}{I_{0}\left(\pi \frac{R_{s}}{L}\right)} I_{0}\left(\pi \frac{r}{L}\right) \cos \left\{\pi \frac{z}{L}\right\}\right), \\
& B_{r}=-B_{0} \frac{1}{I_{0}\left(\pi \frac{R_{s}}{L}\right)} I_{1}\left(\pi \frac{r}{L}\right) \sin \left\{\pi \frac{z}{L}\right\} .
\end{aligned}
$$

The magnetic field is periodic in $z$ with period $2 L$. Here $I_{0}$ and $I_{1}$ are modified Bessel functions and the symbol $R_{s}$ refers to the radius of the separatrix at the midplane in the divertor mirror cell.

Figure 1 shows the magnetic-field axial profile and

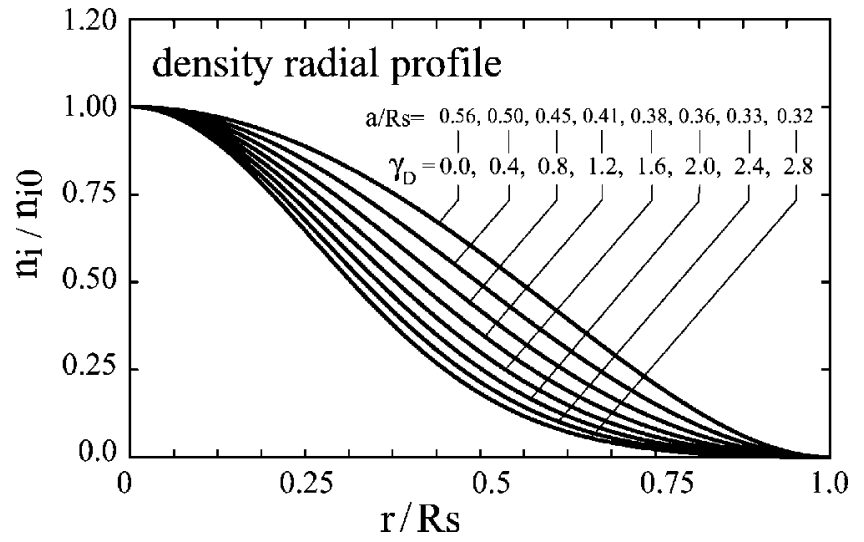

FIG. 2. The radial density profiles given by Eq. (33) for various $\gamma_{D}$. Here $a$ is the half-width of the density, i.e., $n_{i}(a)=0.5 n_{i 0}$.

magnetic-field lines of Eq. (32). In this paper we use $R_{s} / L$ $=0.65$, which gives the mirror ratio $R=2.48$, and assume $B_{0}=3.0 \mathrm{kG}$.

The density and temperature profiles used here are

$$
\begin{aligned}
& n_{i}(\psi)=n_{i 0}\left(1-\frac{\psi}{\psi_{s}}\right) \exp \left\{-\gamma_{D} \frac{\psi}{\psi_{s}}\right\}, \\
& T_{i \perp}(\psi)=T_{i \perp 0}\left(1-\alpha_{T} \frac{\psi}{\psi_{s}}\right),
\end{aligned}
$$

where density and temperature profiles have the magnitude of zero at the magnetic null $\psi=\psi_{s}$.

At first the calculation is carried out for the density profiles given in Fig. 2. Here ion temperature is assumed to be uniform radially (i.e., $\alpha_{T}=0$ ) and to be isotropic $T_{i \perp}=T_{i \|}$. The symbol $a$ in Fig. 2 means the half-width of ion density; that is, $n_{i}(r=a)=0.5 n_{i}(r=0)$, where $r$ is the radius along the midplane in the divertor mirror cell. Figure 3 shows the stability boundary of $m=-1$ and $m=-2$ modes obtained by solving Eq. (28), which reproduces Fig. 2(b) in Ref. 12. The most dangerous mode is $m=-1$, which is not stabilized by ion finite Larmor radius effect at small $a / R_{s}$. On the other hand, the $m=-2$ mode is stabilized by ion FLR in the range

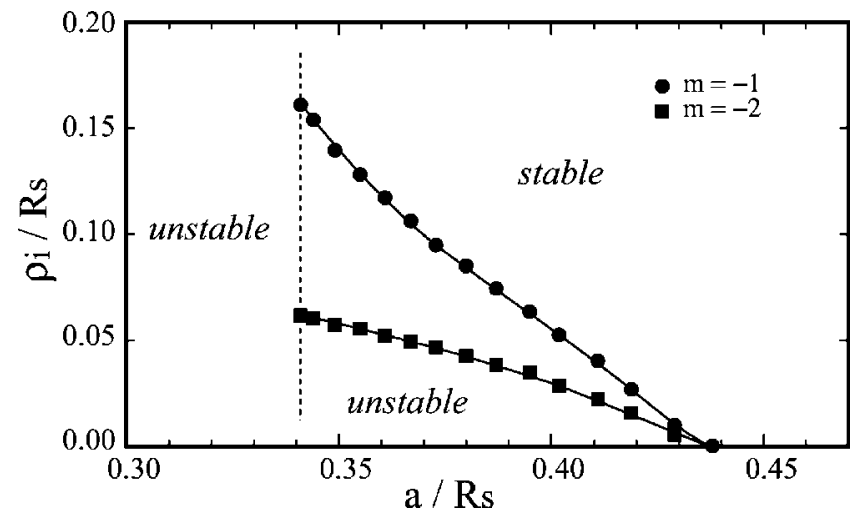

FIG. 3. Stability boundary in the case $T_{i \perp}=T_{i \|}=T_{i 0}$, i.e., uniform and isotropic ion temperatures. Solid circles are the stability boundary for the case $m=-1$ mode and solid squares are the case of $m=-2$. Here $\rho_{i} \equiv\left(T_{i} / M_{i}\right)^{1 / 2} / \omega_{c i}$ and $\omega_{c i} \equiv e B_{0} / M_{i} c$, and $a$ is the half-width of the density, i.e., $n_{i}(a)=0.5 n_{i 0}$. 


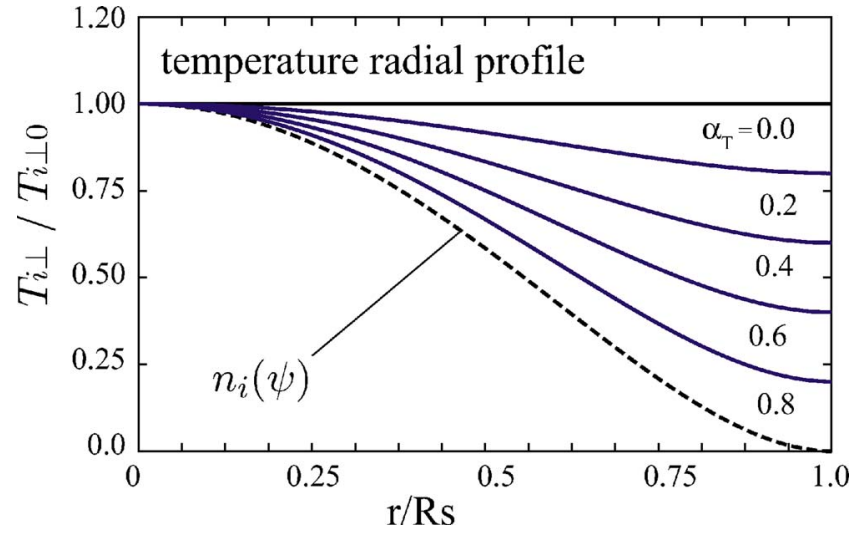

FIG. 4. The radial temperature $T_{i \perp}$ profiles by Eq. (34) for various $\alpha_{T}$. The dashed line is the ion density radial profile for the case of $\gamma_{D}=0$.

of $0.34<a / R_{s}<0.38$ when $\rho_{i} / R_{s} \gtrsim 0.06$. For $a / R_{s} \lesssim 0.35$ no stable regions were found to the $m=-1$ and $m=-2$ modes. Thus for this case, the sharp density profile is not stabilized by the divertor magnetic field. For $a / R_{s} \geqslant 0.44, m=-1$ and $m=-2$, modes are stable even if $\rho_{i} / R_{s}=0$, which indicates that the plasma compressibility in the nonparaxial magnetic field plays a role essential to stability. Therefore, the fat density profile is stabilized by the divertor magnetic field.

We also calculate the effect of the ion temperature gradient on the stability. Figure 4 shows the ion temperature radial profiles used in the calculation given by Eq. (34). Here $\gamma_{D}=0$ is assumed in the density profile of Eq. (33). The stability boundaries, which were determined numerically, are plotted in Fig. 5. As shown in Fig. 3 the $m=-1$ and $m=-2$ flute modes are stable for the case that the density profile with $\gamma_{D}=0$ and $T_{i \perp}=0$. So the stability region exists in the range $\rho_{i} / R_{S} \lesssim 10^{-3}$ in Fig. 5 although the stability boundary is not plotted in the figure. In the actual experiment the parameter $\rho_{i} / R_{S} \lesssim 10^{-3}$ implies very low ion temperature, a regime that is not interesting for the fusion-oriented experiments. ${ }^{19}$ However, the ion Larmor radius $\rho_{i}$ is required to have a length of the order of $R_{s}$ in Fig. 5, in order to stabilize the $m=-1$ and $m=-2$ modes in the case of high ion

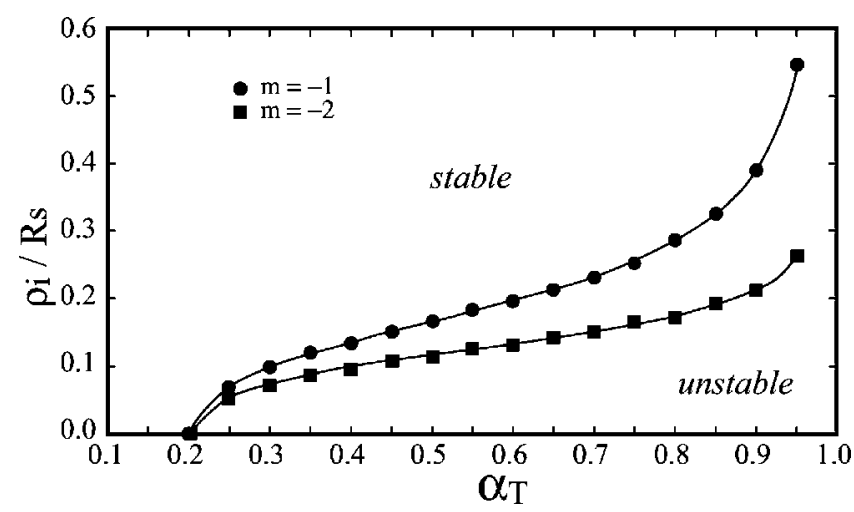

FIG. 5. Stability boundary for various ion temperature radial profiles in the case $\gamma_{D}=0.0$. Here the ion temperature profiles are given in Eq. (34), and isotropic ion temperature $T_{i \perp}=T_{i \|}$ is considered. The parameter $\gamma_{D}=0.0$ gives a density profile with $a / R_{s}=0.56$ shown in Fig. 2 . Solid circles are the stability boundary for the case $m=-1$ mode and solid squares are the case of $m=-2$. temperature. The parameter $\rho_{i} / R_{S} \sim 0.5$ requires extremely high ion temperature, which cannot be achieved in the actual experiments. ${ }^{19}$

Ion density and temperature around the magnetic null are expected to be much lower than those on axis in the experimental device, because the magnetic confinement due to the adiabatic invariance disappears on axis. The numerical results in this paper indicate that such temperature profiles are unstable to the flute modes. However, the results only indicate that the density and ion temperature profiles (33) and (34) are not stable to the flute modes. So one of the remaining problems to be solved is to seek density and temperature profiles that are zero at the magnetic null point and stable to the flute modes.

\section{SUMMARY AND DISCUSSION}

The magnetic divertor can be a candidate for the axisymmetric mirror device stable to the flute modes. In this paper we derived a new equation (28) to investigate flute mode stability in magnetic divertor configuration. This equation includes the effect of nonparaxial magnetic-field lines, ion FLR, the radial dependences of density and ion temperature, and ion temperature anisotropy. Equation (28) reduces to the equation derived by Pastukhov and Sokolov ${ }^{12}$ in the case of uniform, isotropic ion temperature.

One result obtained from Eq. (28), which is shown in Fig. 5, indicates that the divertor model (32) is unstable to the flute modes for the temperature profile with $\alpha_{T}=1$. This result does not mean the divertor model (32) is always unstable to the flute mode, but it indicates that the density (33) and ion temperature (34) profiles used to obtain Fig. 5 are not a compatible choice for the experiment. We adopted the CGL approximation to derive Eq. (28) so that the results obtained by Eq. (28) will yield a necessary but not sufficient condition for stability. It is a useful tool as a first step to design the divertor magnetic-field configuration in a tandem mirror such as Ref. 22.

\section{APPENDIX A: LONGITUDINAL MOTION}

The longitudinal response of electrons to the electrostatic perturbation is given by the electron fluid equation neglecting finite Larmor radius effects. In the following we assume that electrons move quickly and the axial bounce period across plasma is faster than the wave frequency being considered. The perturbed longitudinal electron velocity $v_{e \|}$ and electron density $n_{e 1}$ are given by the equations,

$$
M_{e} n_{e 0} \frac{\partial v_{e \|}}{\partial t}=e n_{e 0} \nabla_{\|} \phi_{1}+e n_{e 1} \nabla_{\|} \phi_{0}-T_{e} \nabla_{\|} n_{e 1},
$$

where we assume that the electron response remains isothermal with respect to the flute perturbation, and

$$
\frac{\partial n_{e 1}}{\partial t}+\nabla \cdot\left(n_{e 0} v_{e \|} \hat{e}_{\|}\right)=0
$$

Here $\hat{e}_{\|}$is the unit vector along a magnetic-field line. Describing the equation in the $(\psi, \theta, \chi)$ coordinates, Eqs. (A1) and (A2) reduce to 


$$
\begin{aligned}
\frac{\partial^{2} n_{e 1}}{\partial t^{2}} & +J \frac{\partial}{\partial \chi}\left(\frac{e n_{e 0}}{M_{e}} \frac{\nabla \chi \cdot \nabla \chi}{J} \frac{\partial \phi_{1}}{\partial \chi}\right) \\
& +J \frac{\partial}{\partial \chi}\left(\frac{e n_{e 1}}{M_{e}} \frac{\nabla \chi \cdot \nabla \chi}{J} \frac{\partial \phi_{0}}{\partial \chi}\right) \\
& -J \frac{\partial}{\partial \chi}\left(\frac{T_{e}}{M_{e}} \frac{\nabla \chi \cdot \nabla \chi}{J} \frac{\partial n_{e 1}}{\partial \chi}\right)=0,
\end{aligned}
$$

where only the electron motion along a magnetic-field line is taken into account. Here $J$ is the Jacobian defined by $J \equiv \nabla \psi \times \nabla \theta \cdot \nabla \chi=B^{2}$. Therefore, electron charge density $\sigma_{e}^{(0)} \equiv-e n_{e 1}$ is given by

$$
\sigma_{e}^{(0)}=-\frac{B^{2}}{4 \pi} \frac{\partial}{\partial \chi}\left(\frac{\omega_{p e}^{2}}{\omega^{2}} \frac{\partial \phi_{1}}{\partial \chi}+\frac{\omega_{p e}^{2}}{\omega^{2}} \frac{n_{e 1}}{n_{e 0}} \frac{\partial \phi_{0}}{\partial \chi}-\frac{\omega_{p e}^{2}}{\omega^{2}} \frac{T_{e}}{e n_{e 0}} \frac{\partial n_{e 1}}{\partial \chi}\right),
$$

where $\omega_{p e}=\left(4 \pi n_{e 0} e^{2} / M_{e}\right)^{1 / 2}$. Noting that the perturbed electron density $n_{e 1}=-\sigma_{e}^{(0)} / e$ appears on the right-hand side of Eq. (A4), the longitudinal electron response function $\hat{K}_{\|}$is determined by solving Eq. (A4),

$$
\sigma_{e}^{(0)}=\hat{K}_{\|}\left[\phi_{1}\right] \text {. }
$$

The second-order electron charge density $\sigma_{e}^{(1)}$ comes from the electron transverse response to the perturbed electrostatic potential. We define the second-order response function $\hat{K}^{(1)}\left[\phi_{1}\right]$, so that $\sigma_{e}^{(1)}+\sigma_{i}=\hat{K}^{(1)}\left[\phi_{1}\right]$, where $\sigma_{i} \equiv e n_{i 1}$ is the perturbed ion charge density. The charge neutrality condition is given by

$$
\sigma_{e}^{(0)}+\sigma_{e}^{(1)}+\sigma_{i}=\hat{K}_{\|}\left[\phi_{1}\right]+\hat{K}^{(1)}\left[\phi_{1}\right]=0 .
$$

We separate the perturbed electrostatic potential as $\phi_{1}=\phi_{1}^{(0)}$ $+\phi_{1}^{(1)}$, where $\phi_{1}^{(0)}$ results from the electron longitudinal response and $\phi_{1}^{(1)}$ comes from the ion and electron transverse response to the electrostatic perturbation $\phi_{1}^{(0)}$ and from electron longitudinal response to $\phi_{1}^{(1)}$.

To the lowest order, the charge neutrality condition (A6) is $\sigma_{e}^{(0)}=\hat{K}_{\|}\left[\phi_{1}^{(0)}\right]=0$, which follows from, using Eq. (A4),

$$
\frac{B^{2}}{4 \pi} \frac{\partial}{\partial \chi}\left(\frac{\omega_{p e}^{2}}{\omega^{2}} \frac{\partial \phi_{1}^{(0)}}{\partial \chi}\right)=0,
$$

which means that $\phi^{(0)}$ is independent of $\chi$ and a function of only $\psi$, i.e., $\phi_{1}^{(0)}=\phi_{1}^{(0)}(\psi)$. This is interesting because even if the electric field along a magnetic-field line exists in equilibrium, a solution for the perturbed electric field in the longitudinal direction that satisfies the charge neutrality condition does not exist to lowest order.

The first order of Eq. (A6) is

$$
\hat{K}_{\|}\left[\phi_{1}^{(1)}\right]+\hat{K}^{(1)}\left[\phi_{1}^{(0)}\right]=0 .
$$

Noting from Eqs. (A4) and (A5) that

$$
\begin{aligned}
\hat{K}_{\|}\left[\phi_{1}^{(1)}\right]= & -\frac{B^{2}}{4 \pi} \frac{\partial}{\partial \chi}\left(\frac{\omega_{p e}^{2}}{\omega^{2}} \frac{\partial \phi_{1}^{(1)}}{\partial \chi}+\frac{\omega_{p e}^{2}}{\omega^{2}} \frac{n_{e 1}}{n_{e 0}} \frac{\partial \phi_{0}}{\partial \chi}\right. \\
& \left.-\frac{\omega_{p e}^{2}}{\omega^{2}} \frac{T_{e}}{e n_{e 0}} \frac{\partial n_{e 1}}{\partial \chi}\right),
\end{aligned}
$$

the first-order Eq. (A8) becomes

$$
\begin{aligned}
\frac{\hat{K}^{(1)}\left[\phi_{1}^{(0)}\right]}{B^{2}}= & \frac{1}{4 \pi} \frac{\partial}{\partial \chi}\left(\frac{\omega_{p e}^{2}}{\omega^{2}} \frac{\partial \phi_{1}^{(1)}}{\partial \chi}-\frac{\omega_{p e}^{2}}{\omega^{2}} \frac{\hat{K}_{\|}\left[\phi_{1}^{(1)}\right]}{e n_{e 0}} \frac{\partial \phi_{0}}{\partial \chi}\right. \\
& \left.+\frac{\omega_{p e}^{2}}{\omega^{2}} \frac{T_{e}}{e^{2} n_{e 0}} \frac{\partial \hat{K}_{\|}\left[\phi_{1}^{(1)}\right]}{\partial \chi}\right) .
\end{aligned}
$$

The condition for the solvability of Eq. (A9) is

$$
\int \frac{\hat{K}^{(1)}\left[\phi_{1}^{(0)}\right]}{B^{2}} \mathrm{~d} \chi=\int \frac{\sigma_{e}^{(1)}+\sigma_{i}}{B^{2}} \mathrm{~d} \chi=0 .
$$

Here the integration is carried out from one end of plasma to the opposite end, and $\omega_{p e}^{2}=0$.

\section{APPENDIX B: ION DISTRIBUTION AT OFF-MIDPLANE}

The equilibrium ion distribution function equation (5) consists of the constants of motion, where local ion velocity components $v_{\|}(\chi, \epsilon, \mu), v_{\perp}(\chi, \epsilon, \mu)$ are a function of the constants of motion through the relation of $\left(M_{i} / 2\right) v_{\perp c c}^{2}=\mu B_{c c}(\psi), \quad\left(M_{i} / 2\right) v_{\| c c}^{2}=\epsilon-\mu B_{c c}(\psi)$. That is, $v_{\perp}(\chi, \epsilon, \mu)=v_{\perp c c} \sqrt{B(\psi, \chi) / B_{c c}(\psi)} \quad$ and $\quad v_{\|}(\chi, \epsilon, \mu)$ $=\sqrt{v_{\| c c}^{2}+v_{\perp c c}^{2}-v_{\perp}^{2}(\chi, \epsilon, \mu)}$. Please note that the ion velocity components $v_{\| c c}, v_{\perp c c}$ at the midplane, as well as $\epsilon$ and $\mu$, are the constants of motion. Therefore, ion distribution $f_{i 0}\left(u_{i}\right)$ in Eq. (6) is written as

$$
\begin{aligned}
f_{i 0}(\psi, \chi) & =n_{i c c}(\psi)\left(\frac{M_{i}}{2 \pi T_{i \perp c c}(\psi)}\right)\left(\frac{M_{i}}{2 \pi T_{i \| c c}(\psi)}\right)^{1 / 2} \exp \left\{-\frac{M_{i} v_{\| c c}^{2}}{2 T_{i \| c c}(\psi)}-\frac{M_{i} v_{\perp c c}^{2}}{2 T_{i \perp c c}(\psi)}\right\} \\
& =n_{i c c}(\psi)\left(\frac{M_{i}}{2 \pi T_{i \perp c c}(\psi)}\right)\left(\frac{M_{i}}{2 \pi T_{i \| c c}(\psi)}\right)^{1 / 2} \exp \left\{-\frac{M_{i}\left(v_{\|}^{2}+v_{\perp}^{2}\right)-M_{i} v_{\perp}^{2} B_{c c}(\psi) / B(\psi, \chi)}{2 T_{i \| c c}(\psi)}-\frac{M_{i} v_{\perp}^{2} B_{c c}(\psi) / B(\psi, \chi)}{2 T_{i \perp c c}(\psi)}\right\} \\
& =n_{i c c}(\psi)\left(\frac{M_{i}}{2 \pi T_{i \perp c c}(\psi)}\right)\left(\frac{M_{i}}{2 \pi T_{i \| c c}(\psi)}\right)^{1 / 2} \exp \left\{-\frac{M_{i} v_{\|}^{2}}{2 T_{i \| c c}(\psi)}-\frac{M_{i} v_{\perp}^{2}}{2}\left(\frac{1}{T_{i \| c c}(\psi)}-\frac{B_{c c}(\psi)}{B(\psi, \chi) T_{i \| c c}(\psi)}+\frac{B_{c c}(\psi)}{B(\psi, \chi) T_{i \perp c c}(\psi)}\right)\right\} \\
& \equiv n_{i}(\psi, \chi)\left(\frac{M_{i}}{2 \pi T_{i \perp}(\psi, \chi)}\right)\left(\frac{M_{i}}{2 \pi T_{i \|}(\psi, \chi)}\right)^{1 / 2} \exp \left\{-\frac{M_{i} v_{\|}^{2}}{2 T_{i \|}(\psi, \chi)}-\frac{M_{i} v_{\perp}^{2}}{2 T_{i \perp}(\psi, \chi)}\right\} .
\end{aligned}
$$


Therefore, a local density $n_{i}(\psi, \chi)$, and local temperatures $T_{i \|}(\psi, \chi), T_{i \perp}(\psi, \chi)$ are described by Eq. (26) in terms of $n_{i c c}(\psi), T_{i \| c c}(\psi), T_{i \perp c c}(\psi)$.

\section{APPENDIX C: COEFFICIENTS $\boldsymbol{H}_{i, j}$ IN EQ. (28)}

Coefficients $H_{i, j}$ used in Eqs. (28) are given by the following equation:

$$
\begin{aligned}
& H_{1,1}=\int \frac{r^{2}}{B^{2}} h_{\text {local }} \mathrm{d} \chi \quad: H_{1,2}=\int \frac{r^{2}}{B^{2}} h_{\text {local }}^{2} \mathrm{~d} \chi \\
& H_{1,3}=\int \frac{r^{2}}{B^{2}} \frac{\partial h_{\text {local }}^{2}}{\partial \psi} \mathrm{d} \chi \quad \text { : } \\
& H_{2,1}=\int \frac{1}{r^{2} B^{4}} h_{\text {local }} \mathrm{d} \chi \quad: H_{2,2}=\int \frac{1}{r^{2} B^{4}} h_{\text {local }}^{2} \mathrm{~d} \chi \\
& H_{2,3}=\int \frac{1}{r^{2} B^{4}} \frac{\partial h_{\text {local }}^{2}}{\partial \psi} \mathrm{d} \chi \quad \text { : } \\
& H_{3,1}=\int \frac{r^{2}}{B^{3}} \frac{\partial B}{\partial \psi} h_{\text {local }} \mathrm{d} \chi \quad: H_{3,2}=\int \frac{r^{2}}{B^{3}} \frac{\partial B}{\partial \psi} h_{\text {local }}^{2} \mathrm{~d} \chi \\
& H_{3,3}=\int \frac{r^{2}}{B^{3}} \frac{\partial B}{\partial \psi} \frac{\partial h_{\text {local }}^{2}}{\partial \psi} \mathrm{d} \chi \quad: \quad H_{3,4}=\int \frac{r^{2}}{B^{3}} \frac{\partial B}{\partial \psi} \frac{\partial h_{\text {local }}}{\partial \psi} \mathrm{d} \chi \\
& H_{3,5}=\int \frac{r^{2}}{B^{3}} \frac{\partial B}{\partial \psi} \mathrm{d} \chi \\
& H_{4,1}=\int h_{\text {local }}^{2} \frac{\partial}{\partial \psi}\left(\frac{r^{2}}{B^{2}}\right) \mathrm{d} \chi \quad: \quad H_{4,2}=\int \frac{\partial h_{\text {local }}^{2}}{\partial \psi} \frac{\partial}{\partial \psi}\left(\frac{r^{2}}{B^{2}}\right) \mathrm{d} \chi \\
& H_{4,3}=\int \frac{\partial^{2} h_{\text {local }}^{2}}{\partial \psi^{2}} \frac{\partial}{\partial \psi}\left(\frac{r^{2}}{B^{2}}\right) \mathrm{d} \chi \quad: \\
& H_{5,1}=\int \frac{1}{B^{3}} \frac{\partial B}{\partial \psi} h_{\text {local }} \mathrm{d} \chi \quad: H_{5,2}=\int \frac{1}{B^{3}} \frac{\partial B}{\partial \psi} h_{\text {local }}^{2} \mathrm{~d} \chi \\
& H_{5,3}=\int \frac{1}{B^{3}} \frac{\partial B}{\partial \psi} \frac{\partial h_{\text {local }}^{2}}{\partial \psi} \mathrm{d} \chi \quad: \quad H_{5,4}=\int \frac{1}{B^{3}} \frac{\partial B}{\partial \psi} \frac{\partial h_{\text {local }}}{\partial \psi} \mathrm{d} \chi \\
& H_{5,5}=\int \frac{1}{B^{3}} \frac{\partial B}{\partial \psi} \mathrm{d} \chi \\
& H_{6,1}=\int \frac{1}{B^{4}}\left(\frac{\partial B}{\partial \psi}\right)^{2} h_{\text {local }} \mathrm{d} \chi \quad: \quad H_{6,2}=\int \frac{1}{B^{4}}\left(\frac{\partial B}{\partial \psi}\right)^{2} h_{\text {local }}^{2} \mathrm{~d} \chi \\
& H_{6,3}=\int \frac{1}{B^{4}}\left(\frac{\partial B}{\partial \psi}\right)^{2} \frac{\partial h_{\text {local }}^{2}}{\partial \psi} \mathrm{d} \chi: H_{6,4}=\int \frac{1}{B^{4}}\left(\frac{\partial B}{\partial \psi}\right)^{2} \frac{\partial h_{\text {local }}^{3}}{\partial \psi} \mathrm{d} \chi \\
& H_{6,5}=\int \frac{1}{B^{4}}\left(\frac{\partial B}{\partial \psi}\right)^{2} \mathrm{~d} \chi \quad: H_{6,6}=\int \frac{1}{B^{4}}\left(\frac{\partial B}{\partial \psi}\right)^{2} h_{\text {local }}^{3} \mathrm{~d} \chi .
\end{aligned}
$$

${ }^{1}$ G. I. Dimov, V. V. Zakaidakov, and M. E. Kishinevskij, Sov. J. Plasma Phys. 2, 326 (1976).

${ }^{2}$ T. K. Fowler and B. G. Logan, Comments Plasma Phys. Controlled Fusion 2, 167 (1977).

${ }^{3}$ R. H. Cohen, Nucl. Fusion 19, 1579 (1979).

${ }^{4}$ D. D. Ryutov and G. V. Stupakov, Sov. J. Plasma Phys. 4, 278 (1978).

${ }^{5}$ D. D. Ryutov and G. V. Stupakov, Sov. Phys. Dokl. 23, 412 (1978).

${ }^{6}$ I. Katanuma, Y. Kiwamoto, S. Adachi, M. Inutake, K. Ishii, K. Yatsu, K. Sawada, and S. Miyoshi, Nucl. Fusion 27, 2041 (1987).

${ }^{7}$ I. Katanuma, Y. Kiwamoto, K. Ishii, K. Yatsu, and S. Miyoshi, Phys. Fluids B 1, 1459 (1989).

${ }^{8}$ J. R. Myra and P. J. Catto, Phys. Rev. Lett. 48, 620 (1982).

${ }^{9}$ T. Ito and I. Katanuma, Phys. Plasmas 12, 082512 (2005).

${ }^{10}$ D. D. Ryutov and G. V. Stupakov, Sov. J. Plasma Phys. 12, 815 (1986).

${ }^{11}$ B. Lane, R. S. Post, and J. Kesner, Nucl. Fusion 27, 277 (1987).

${ }^{12}$ V. P. Pastukhov and A. Yu. Sokolov, Fiz. Plazmy 17, 1043 (1991) [Sov. J. Plasma Phys. 17, 603 (1991)].

${ }^{13}$ G. Chew, M. Goldberger, and F. Low, Proc. R. Soc. London A236, 112 (1956).

${ }^{14}$ I. Katanuma, T. Ito, H. Saimaru, Y. Sasagawa, V. P. Pastukhov, K. Ishii, Y. Tatematsu, T. Saito, Md. K. Islam, Y. Nakashima, and T. Cho, Proceedings of the Fifth International Conference on Open Magnetic Systems for Plasma Confinement, Novosibirsk, Russia, 5-9 July 2004, edited by N. A. Uckan [Trans. Fus. Sci. Tech. 47, 276 (2005)].

${ }^{15}$ V. V. Arsenin and A. Yu. Kuyanov, Fiz. Plazmy 26, 793 (2000) [Plasma Phys. Rep. 26, 741 (2000)].

${ }^{16}$ V. V. Arsenin and A. Yu. Kuyanov, Fiz. Plazmy 27, 675 (2001) [Plasma Phys. Rep. 27, 635 (2001)].

${ }^{17}$ J. A. Casey, B. G. Lane, J. H. Irby, K. L. Brau, S. N. Golovato, W. C. Guss, J. Kesner, R. S. Post, E. Sevillano, and J. Zielinski, Phys. Fluids 31, 2009 (1988).

${ }^{18}$ Y. Yasaka, A. Maruyama, and N. Takano, Proceedings of the Fourth International Conference on Open Magnetic Systems for Plasma Confinement, Jeju Island, Korea, 1-4 July 2003, edited by N. A. Uckan [Trans. Fus. Sci. Tech. 43, 44 (2003)].

${ }^{19}$ T. Cho, M. Yoshida, J. Kohagura, M. Hirata, T. Numakura, H. Higaki, H. Hojo, M. Ichimura, K. Ishii, K. Md. Islam, A. Itakura, I. Katanuma, Y. Nakashima, T. Saito, Y. Tatematsu, M. Yoshikawa, Y. Kojima, S. Tokioka, N. Yokoyama, Y. Tomii, T. Imai, V. P. Pastukhov, S. Miyoshi, and GAMMA10 Group, Phys. Rev. Lett. 94, 085002-1 (2005).

${ }^{20}$ T. B. Kaiser, W. M. Nevins, and L. D. Pearlstein, Phys. Fluids 26, 351 (1983).

${ }^{21}$ I. Katanuma, Y. Tatematsu, K. Ishii, T. Tamano, and K. Yatsu, J. Phys. Soc. Jpn. 69, 3244 (2000).

${ }^{22}$ I. Katanuma, Y. Sasagawa, Y. Tatematsu, Y. Nakashima, T. Cho, and V. P. Pastukhov, Nucl. Fusion 46, 608 (2006). 